\title{
LncRNA prostate androgen-regulated transcript 1 (PART 1) functions as an oncogene in osteosarcoma via sponging miR-20b-5p to upregulate BAMBI
}

\author{
Zhimin Pan ${ }^{1 \#}$, Fengbo Mo ${ }^{1 \#}$, Hucheng Liu ${ }^{1 \#}$, Jin Zeng ${ }^{1}$, Kai Huang ${ }^{2}$, Sheng Huang ${ }^{1}$, Zhiyou Cao ${ }^{1}$, \\ Xiaoyu Xu ${ }^{1}$, Jianyun Xu ${ }^{1}$, Tianmi Liu ${ }^{3}$, Jiang Huang ${ }^{1}$ \\ ${ }^{1}$ Department of Orthopaedics, The First Affiliated Hospital of Nanchang University, Nanchang, China; ${ }^{2}$ Department of Orthopedics, Zhabei Central \\ Hospital of Jing'an District, Shanghai, China; ${ }^{3}$ Department of Rehabilitation, The First Affiliated Hospital of Nanchang University, Nanchang, \\ China \\ Contributions: (I) Conception and design: J Huang, T Liu; (II) Administrative support: F Mo; (III) Provision of study materials or patients: Z Pan, F \\ Mo; (IV) Collection and assembly of data: Z Pan, H Liu, J Zeng; (V) Data analysis and interpretation: H Liu, K Huang, S Huang; (VI) Manuscript \\ writing: All authors; (VII) Final approval of manuscript: All authors. \\ \#These authors contributed equally to this work and are first co-authors. \\ Correspondence to: Jiang Huang, MD. Department of Orthopaedics, The First Affiliated Hospital of Nanchang University, 17 Yongwai St., Nanchang \\ 330006, China. Email: huangjiang4680@hotmail.com; Tianmi Liu, MD. Department of Rehabilitation, The First Affiliated Hospital of Nanchang \\ University, No.17, Yongwai St., Nanchang 330006, China. Email: 3337234848@qq.com.
}

Background: Osteosarcoma (OS) is an aggressive bone cancer that most commonly affects adolescents and children. Emerging studies have shown that long noncoding RNA (lncRNA) performs essential roles in the occurrence and development of many tumors. Prostate androgen-regulated transcript 1 (PART 1) has been reported as a tumor oncogene; despite this, the mechanisms underlying its involvement in OS are unclear.

Methods: OS and paired normal tissue samples were obtained, and gene expressions were detected by real time-quantitative polymerase chain reaction (RT-qPCR). The functions of PART 1 in OS cell proliferation, invasion, and migration were determined by Cell Counting Kit-8 (CCK-8) and Transwell assays. Furthermore, the binding sites of PART 1 and miR-20b-5p as well as those between miR-20b-5p and bone morphogenic protein and activin membrane-bound inhibitor homolog (BAMBI) were verified by bioinformatics analysis and dual-luciferase reporter assay.

Results: Our study found obvious overexpression of PART 1 in OS tissues and cells. Furthermore, PART 1 overexpression facilitated OS cell proliferation, invasion, and migration. Further mechanistic investigations revealed that PART 1 could sponge to miR-20b-5p, which was expressed at a low level in OS tissues and cells. Importantly, miR-20b-5p overexpression inhibited OS cell proliferation, invasion, and migration. Additionally, BAMBI was confirmed as a downstream gene of miR-20b-5p, and its expression was reversely modulated by miR-20b-5p and positively modulated by PART 1 . Rescue experiments suggested that BAMBI was involved in PART 1-mediated promotion of OS progression.

Conclusions: PART 1 serves as a competing endogenous RNA to promote OS tumorigenesis via its regulation of the miR-20b-5p/BAMBI axis, which may provide a promising therapeutic biomarkers for OS patients.

Keywords: Osteosarcoma (OS), prostate androgen-regulated transcript 1 (PART 1), miR-20b-5p, BAMBI

Submitted Jan 19, 2021. Accepted for publication Mar 11, 2021.

doi: $10.21037 / \mathrm{atm}-21-658$

View this article at: http://dx.doi.org/10.21037/atm-21-658 


\section{Introduction}

Osteosarcoma (OS) is a bone malignancy originating from osteoid bone tissue which has a high mortality rate $(1,2)$. Despite the effectiveness of therapeutic modalities for OS, including surgical resection, neoadjuvant, or adjuvant radiotherapy or chemotherapy, the rates of mortality and metastasis among patients with the disease are still extremely high. Moreover, for patients with metastatic or recurrent disease, the survival outlook is dismal $(3,4)$. Multiple pathophysiological and pathological processes, such as epithelial-mesenchymal transition (EMT), drug resistance, autophagy, and the invasion, migration, apoptosis, and proliferation of OS cells are closely related to OS development (5-7). Unfortunately, the mechanism underlying OS progression has not been fully uncovered. Therefore, determining the key molecules implicated in OS may prove helpful to efforts to develop effective prevention and treatment measures.

Recently, a new series of noncoding RNAs (ncRNAs) have been found, including circular RNAs (circRNAs), long ncRNAs (lncRNAs, RNA transcripts $>200$ bp in length) and microRNAs (miRNAs, RNA transcripts $\sim 22$ bp in length), all of which have important effects on tumor development and the modulation of basic protein effectors of cellular functions $(8,9)$. As the 2 main members of the ncRNA family, lncRNAs and miRNAs play pivotal roles in OS tumorigenesis.

An increasing bank of evidence has confirmed that numerous lncRNAs play key roles in multiple pathological and physiological cellular processes, including cell invasion, differentiation, apoptosis, and proliferation (10). Dysregulation of lncRNA expression has been found to have oncogenic effects (e.g., PROX1-AS1 in prostate cancer and NCK1-AS1 in urinary bladder cancer) $(11,12)$ or tumor suppressive effects (e.g., TSLNC8 in breast cancer and RP11-422N16.3 in hepatocellular carcinoma) $(13,14)$ during carcinogenesis. So far, a number of lncRNAs have been reported to possess promising prognostic or diagnostic value for OS $(15,16)$; however, the role of prostate androgen-regulated transcript 1 (PART 1) in this malignancy is largely unknown. Recently, studies by showed that PART1 regulated the apoptosis of chondrocytes in osteoarthritis (17). A recent study by investigated the functions of PART1 in hepatocellular carcinoma and found that PART1 served as oncogenic lncRNA through sponging miR-590-3p to upregulate HMGB2 expression in hepatocellular carcinoma (18). Accordingly, we hypothesize that PART1 may play a key role in OS development.

In recent decades, miRNAs have also been found to 50 serve as epigenetic regulators in disease development. MiRNAs repress gene expression and participate in gene silencing via direct interaction with the 3 '-untranslated region (UTR) of target messenger RNAs (mRNAs), leading to the repression of mRNA translation or degradation (19). miRNAs participate in a variety of pathological and biological processes, including carcinogenesis, metabolism, and embryonic development (20). Several crucial activities of miRNAs in OS have been reported (21). Specifically, lncRNAs have been demonstrated to be endogenously competing RNAs which target miRNAs to inhibit miRNA-associated gene degradation.

In the present study, we investigated the expression and roles of PART 1 in OS, as well as the potential underlying regulatory mechanism. We elucidated that PART 1 serves as a competing endogenous RNA in OS by sponging miR20b-5p. We present the following article in accordance with the MDAR reporting checklist (available at http://dx.doi. org/10.21037/atm-21-658).

\section{Methods}

\section{Tissue samples}

Forty-six pairs of OS tissue samples and matched noncancerous tissues were harvested from patients who underwent excision surgery for OS in our hospital. All of the patients were radiation and chemotherapy naive. Liquid nitrogen was used to freeze the tissue samples before the extraction of total RNA. All patients signed a written informed consent form. All procedures in our study were carried out in accordance with the Helsinki Declaration (as revised in 2013). The study was approved by the Ethics Committee Board of our Hospital.

\section{Cell lines and cell culture}

OS cell lines [HOS (TCHu167) and MG-63 (TCHu124)] and human fetal osteoblastic cell line (hFOB) 1.19 were acquired from the Type Culture Collection of the Chinese Academy of Sciences (Shanghai, China). Cells were maintained in Dulbecco's Modified Eagle Medium (DMEM; Invitrogen, Carlsbad, CA, USA) with 10\% fetal bovine serum (FBS; Invitrogen) in a humidified chamber containing $5 \% \mathrm{CO}_{2}$ at $37{ }^{\circ} \mathrm{C}$. 


\section{Cell transfection}

The miR-20b-5p inhibitor and mimic were designed by Gene Pharma (Shanghai, China). The whole sequences of PART 1 and bone morphogenic protein and activin membrane-bound inhibitor homolog (BAMBI) were cloned into pcDNA3.1 vector to overexpress PART 1 and BAMBI, respectively. For knockdown of PART 1, its small interfering RNAs (siRNAs) were synthesized as si-PART 1 by Gene Pharma (Shanghai, China). Lipofectamine 2000 (Invitrogen) was employed to transfect the above plasmids into HOS and MG-63 cells.

\section{Real time-quantitative polymerase chain reaction (RT- qPCR) assay}

TRIzol reagent (Invitrogen) was utilized for the extraction of total RNA from OS tissue samples and cultured cell lines, afterwards, a reverse transcription reaction was performed using a reverse transcription kit (Takara Bio Company, Shiga, Japan). qRT-PCR was completed with SYBR ${ }^{\circledR}$ Green PCR Master mix (Thermo Fisher Scientific, Inc., MA, USA) on an ABI Prism 7500 Sequence Detection system (Applied Biosystems; Thermo Fisher Scientific, Inc.) in adherence to the manufacturers' protocols and with U6 or GAPDH serving as an internal control. The $2^{-\Delta \Delta C t}$ method was used for measurement of relative gene expressions. The primers used were as follows: PART1 Forward, 5'-AAG GCC GTG TCA GAA CTC AA-3' and Reverse, 5'-GTT TTC CAT CTCA GCC TGG A-3'; miR-20b-5p forward, 5'-ACA CTC CAG CTG GGC AAA GTG CTC ATA GT-3' and reverse, 5'-TGG TGT CGT GGA GTC G-3'; BAMBI forward, 5'-CTC AAA TTC CCC ACT CAC CCA-3' and reverse, 5'-GCT GAT ACC TGT TTC CTT GTC CTG-3'; U6 forward, 5'-CTC GCT TCG GCA GCA CA-3' and reverse, 5'-AAC GCT TCA CGA ATT TGC GT-3'; GAPDH forward, 5'-AAT CCC ATC ACC ATC TTC CA-3' and reverse, 5'-TGG ACT CCA CGA CGT ACT CA-3'.

\section{Cell Counting Kit-8 (CCK-8) assay}

To determine cell viability, a CCK-8 assay was carried out as instructed by the manufacturer. OS cells were inserted into a 96-well plate and harvested at 24, 48, 72 or 96 hours post transfection. Then, after the indicated amount of time, CCK- 8 was added to each well and the cells were incubated for a further 1 hour. The absorbance was detected at
$450 \mathrm{~nm}$ using a microplate reader (Bio-Rad Laboratories, 145 Hercules, CA, USA).

\section{Transwell assay}

The migration and invasion abilities of cells were detected by Transwell assay. Cell migration ability was determined using $6.5-\mathrm{mm}$ Transwell chambers $(8.0 \mu \mathrm{m}$ pore size; BD Biosciences, Franklin Lakes, NJ, USA), and cell invasion ability was assessed using Transwell chambers precoated with Matrigel (BD Biosciences). Briefly, OS cells were resuspended in serum-free medium and then seeded into the apical chambers, with the bottom chambers filled with DMEM containing $10 \%$ FBS. After 24 hours of incubation, non-invasive or non-migratory cells were removed with cotton swabs. Cells located in the lower chamber were fixed and stained. Finally, cells in 5 randomly selected visual fields were quantified under a light microscope (Olympus Corp., Tokyo, Japan).

\section{Western blot}

Total protein extraction was accomplished using RIPA buffer (Beyotime, Shanghai, China). After measurement of the protein concentration using a bicinchoninic acid protein assay kit (Beyotime), the protein samples were subjected to sodium dodecyl sulfate-polyacrylamide gel electrophoresis (SDS-PAGE) separation and transferred onto a polyvinylidene difluoride (PVDF) membrane. The membrane was blocked with 5\% skim milk and incubated with specific primary antibodies against BAMBI (ab203070; 1:1,000, Abcam, Cambridge, MA, USA) and GAPDH (ab9485; 1:2000, Abcam, Cambridge, MA, USA) at $4{ }^{\circ} \mathrm{C}$. After incubation overnight, the membrane was incubated with horseradish peroxidase (HRP)-conjugated goat antirabbit (1:2,000, Abcam, Cambridge, MA, USA) secondary antibody for 2 hours at room temperature. Finally, the signals were detected using an electrochemiluminescence (ECL) kit (Thermo Fisher Scientific, Inc.). GAPDH was used as the internal control.

\section{Luciferase reporter assay}

Dual-luciferase reporter assay (Promega, Madison, WI, USA) was performed to verify the relationships between PART1 or BAMBI and miR-20b-5p. After that, the wildtype PART1 and mutant PART1 sequences harboring predicted miR-20b-5p binding sites were synthesized and 
Page 4 of 13

A

B

C
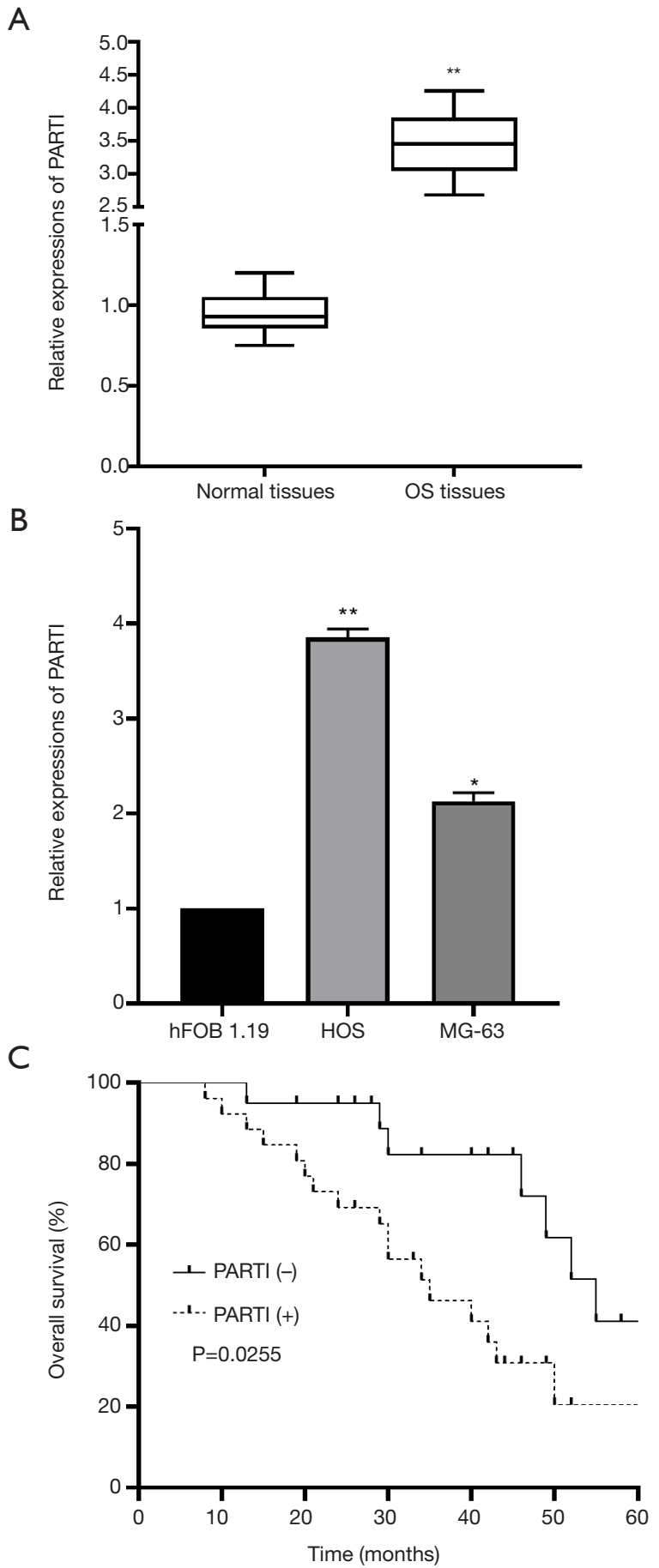

Figure 1 Overexpression of PART 1 in OS indicated shorter overall survival. $(\mathrm{A}, \mathrm{B})$ real time-quantitative polymerase chain reaction (RT-qPCR) analysis demonstrated that PART 1 expression was upregulated in OS tissues and cells. (C) High PART 1 expression was associated with shorter overall survival in OS patients. OS, osteosarcoma; PART 1, prostate androgen-regulated transcript $1 .{ }^{*}, \mathrm{P}<0.05 ;{ }^{* *}, \mathrm{P}<0.01$. inserted into the pGL3-control vector (Promega, Madison, WI, USA) to construct the luciferase reporter vector of PART1-WT and PART1-Mut. Similarly, the wild-type BAMBI 3 '-untranslated regions (UTR) and mutant BAMBI 3'-UTR sequences containing embracing predicted miR$20 \mathrm{~b}-5 \mathrm{p}$ binding sites were synthesized and inserted into the pGL3-control vector for the construction of the luciferase reporter vectors of BAMBI-WT and BAMBIMut. Following this, the luciferase reporter vectors were cotransfected into OS cells with NC-mimics or miR-20b5 p mimics using Lipofectamine 2000 (Invitrogen) for the execution of the dual-luciferase reporter assay, respectively. Finally, cells were harvested in 48 hours post transfection and the luciferase activities of luciferase reporter vectors were evaluated via the dual-luciferase reporter assay kit (Promega).

\section{Statistical analysis}

All of the above experiments were performed in triplicate. SPSS 17.0 version (SPSS Inc., Chicago, IL, USA) was used to perform the statistical analyses. Data were tested using Student's t-test or one-way analysis of variance with Tukey's post-hoc test. The relationships between the expressions of miR-20b-5p and PART 1, PART 1 and BAMBI were assessed by Spearman's or Pearson's correlation analysis. The overall survival of the OS patients was determined with Kaplan-Meier curve together with log-rank test. $\mathrm{P}<0.05$ was considered to indicate significant difference.

\section{Results}

\section{High PART 1 expression in OS tissue indicated a poor prognosis}

To determine the clinical significance of PART 1 in OS, we firstly detected the expression level of PART 1 in OS tissues and matched non-tumor tissues. The RT-qPCR results demonstrated that PART 1 expression was significantly increased in OS tissues compared to non-tumor tissues (Figure 1A). Similarly, upregulation of PART 1 was also observed in OS cells (Figure $1 B$ ). The survival analysis indicated that OS patients with PART 1 upregulation had strikingly shorter overall survival compared to the patients with lower PART 1 expression (Figure 1C). Overall, these results showed that PART 1 was upregulated in patients with OS and indicated a poor prognosis. 


\section{PART 1 accelerated OS cell proliferation, invasion, and migration}

Having detected the aberrant up-regulation of PART 1 in OS tissues, we next performed functional assays, including a CCK-8 assay and Transwell assays, to determine the functions of PART 1 in the progression of OS. The cell lines MG-63 and HOS were transfected with pcDNA3.1PART 1 or si-PART 1 . The results of RT-qPCR verified that PART 1 was successfully overexpressed in MG63 cells and was knocked down in HOS cells following transfection with pcDNA3.1-PART 1 or si-PART 1 (Figure 2A). The CCK-8 assay showed that pcDNA3.1PART 1 significantly elevated the viability of MG-63 cells, whereas the proliferative ability of HOS cells was obviously reduced by si-PART 1 transfection (Figure $2 A$ ). Also, the Transwell assays revealed that PART 1 upregulation promoted the migration and invasion abilities of MG-63 cells (Figure 2B). In contrast, PART 1 knockdown notably reduced HOS cell migration and invasion (Figure 2B). Taken together, these observations suggested that PART 1 upregulation contributed to the malignant progression of OS.

\section{PART 1 acted as a sponge of miR-20b-5p in OS cells}

To determine the molecular mechanisms participating in PART 1-mediated OS progression, miRNAs could potentially serve as targets for PART 1 were predicted with Starbase. Results showed that PART 1 contained conserved binding sites for miR-20b-5p (Figure $3 A$ ). Subsequently, a luciferase reporter assay was performed to verify the correlation of PART 1 with miR-20b-5p. The miR-20b-5p mimics noticeably decreased the luciferase activities of the PART 1-wt plasmid; however, we failed to observe a notable difference in the luciferase activities of the PART 1-mut plasmid (Figure 3B). Next, the expression levels of miR20b-5p in cells transfected with pcDNA3.1-PART 1 or siPART 1 were measured by RT-qPCR. When PART 1 was overexpressed, miR-20b-5p expression was decreased, while PART 1 knockdown dramatically increased miR-20b-5p expression (Figure 3C). Similarly, the regulatory functions of miR-20b-5p in PART 1 expression were also investigated. As shown in Figure 3D, miR-20b-5p inhibition resulted in significant upregulation of PART 1 , whereas the opposite effect was observed with miR-20b-5p overexpression. Additionally, in OS tissues, a significant decrease in miR20b-5p expression was detected (Figure 3E), and a negative correlation between the expressions of PART 1 and miR20b-5p was also confirmed (Figure 3F).

288

$m i R-20 b-5 p$ inbibited proliferation, invasion and migration in OS cells

The expression levels of miR-20b-5p in OS cells were further analyzed. A remarkable decrease in miR-20b-5p in OS cells was verified (Figure 4A). HOS and MG-63 cells were transfected with miR-20b-5p mimics or inhibitor to induce miR-20b-5p overexpression or inhibition, respectively. The transfection was confirmed to have been successfully completed by RT-qPCR (Figure 4B). The regulatory effects of miR-20b-5p on OS cell proliferation, invasion, and migration were subsequently investigated. The results showed that miR-20b-5p overexpression inhibited OS cell proliferation, invasion, and migration, while miR-20b-5p silencing exerted the opposite functions (Figure 4C,D).

\section{BAMBI served as a target of miR-20b-5p in LAC cells}

We further explored the mechanism underlying the promotion of OS progression by the PART 1/miR-20b$5 \mathrm{p}$ axis. TargetScan showed that BAMBI contained binding sites of miR-20b-5p (Figure $5 A$ ). The direct binding of miR-20b-5p to the 3'-UTR of BAMBI at putative sites was confirmed by the results of a luciferase reporter assay (Figure 5B). BAMBI was significantly inhibited by miR-20b-5p overexpression and promoted by miR-20b$5 \mathrm{p}$ inhibition (Figure $5 C, D$ ). Furthermore, BAMBI was markedly upregulated in OS tissue samples compared to the para-carcinoma tissues (Figure 5E). In addition, a positive correlation of the expressions of BAMBI and PART 1 was found to exist in OS tissues (Figure 5F).

\section{PART 1 promoted OS tumorigenesis by sponging miR- 20b-5p to upregulate BAMBI}

To determine whether the miR-20b-5p/BAMBI axis was implicated in the oncogenic functions of PART 1 in OS cells, a rescue assay was carried out. MiR-20b-5p inhibitor and pcDNA-BAMBI were transfected into OS cells together with si-PART 1. As shown in Figure 6A, BAMBI expression was significantly downregulated by si-PART 1 , and this reduction was PART 1 ally reversed by silencing of miR-20b-5p or BAMBI overexpression. Additionally, we found that the suppressive effects of PART 1 knockdown 


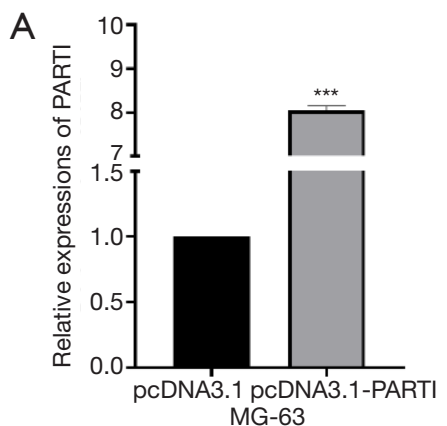

B

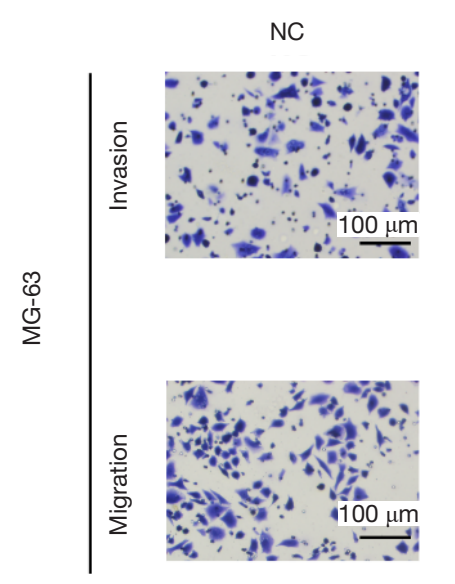

pcDNA3.1
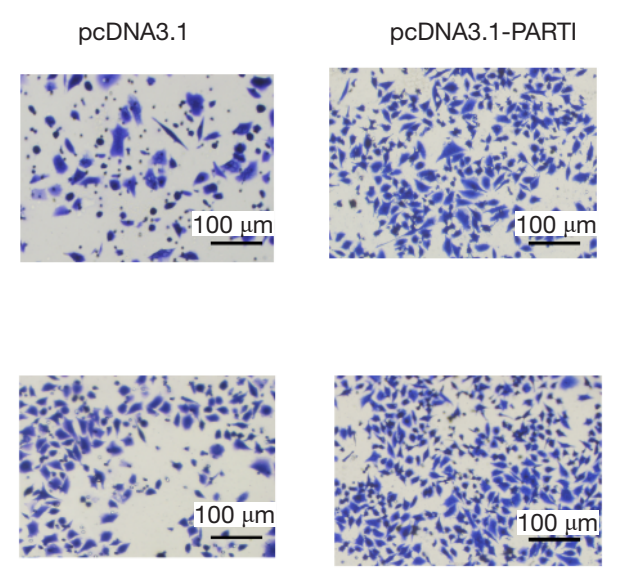

NC
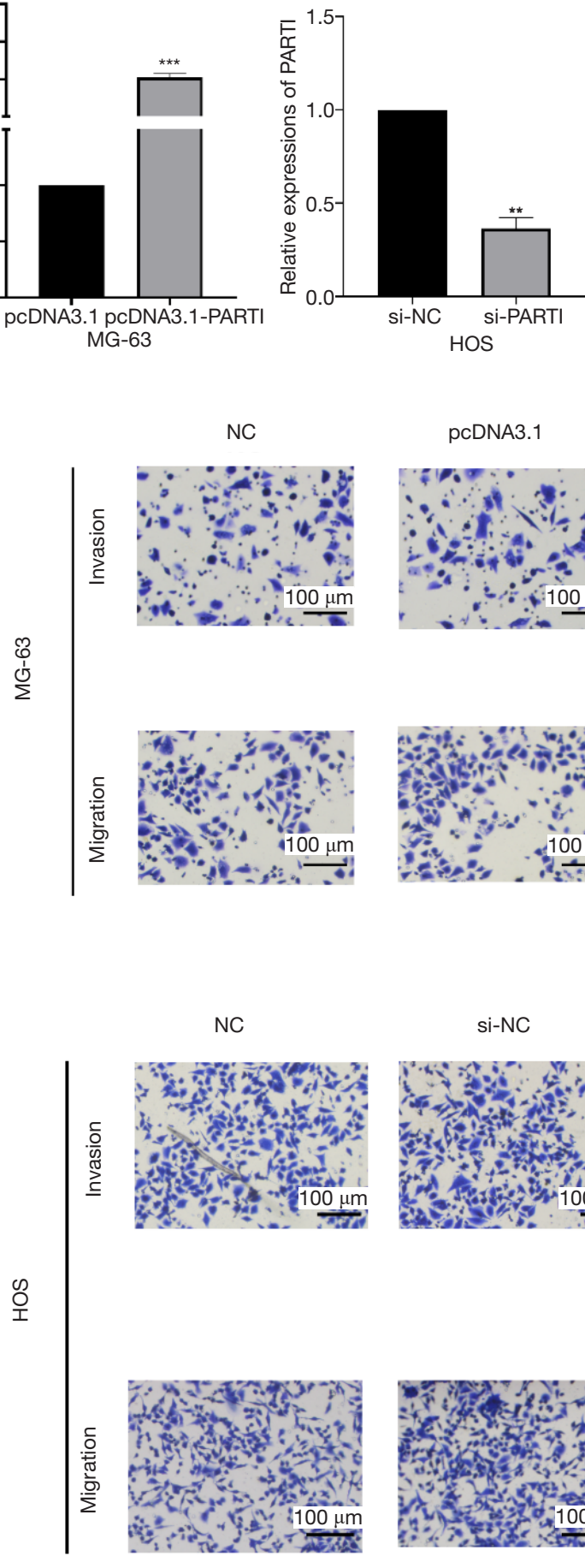

si-NC
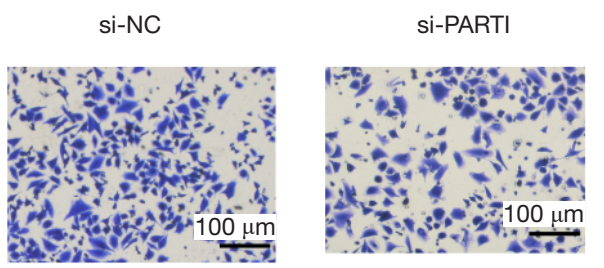
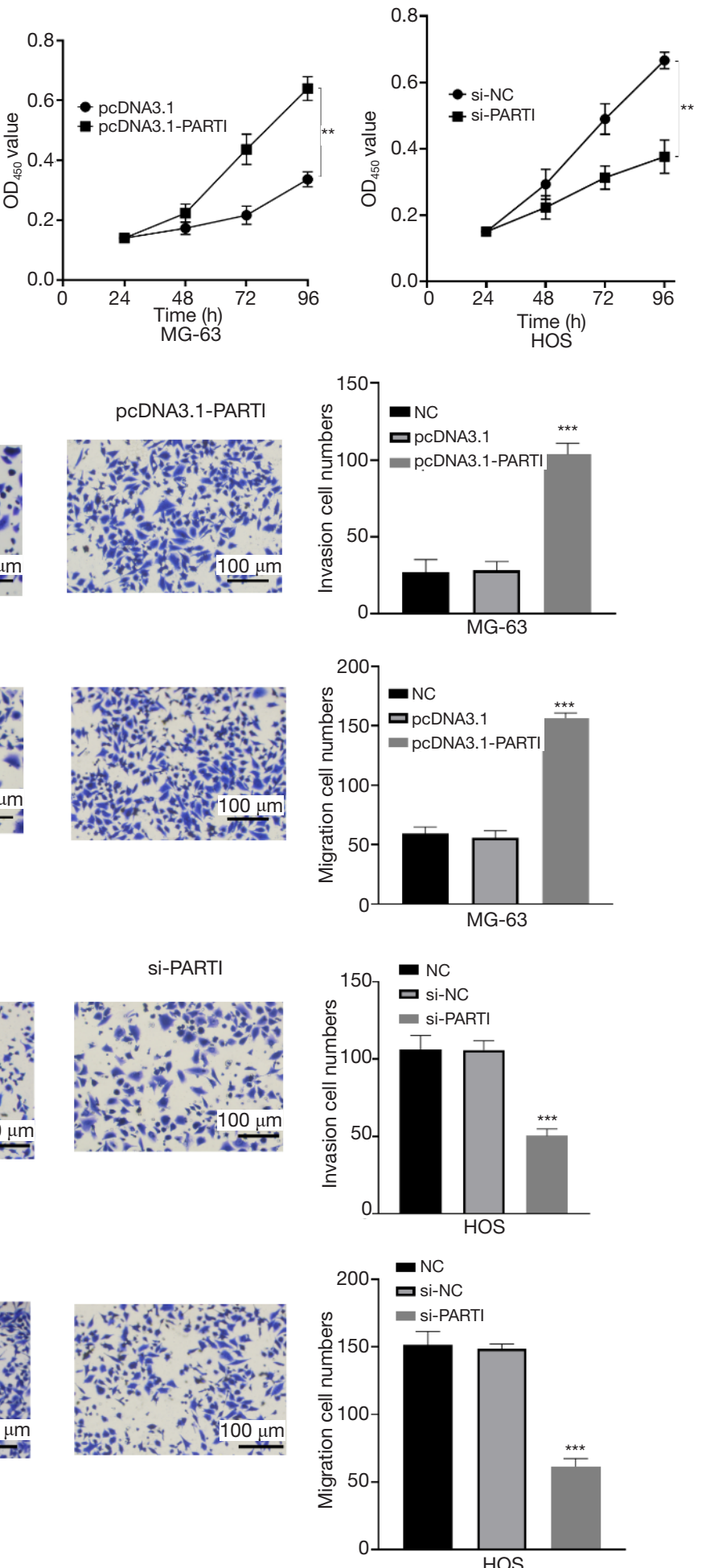

Figure 2 PART 1 accelerated OS cell proliferation, invasion, and migration. (A) Successful upregulation or downregulation of PART 1 in OS cells was confirmed by RT-qPCR and upregulation promoted OS cell viability, as demonstrated by Cell Counting Kit- 8 assay. (B) Transwell assays indicated that PART 1 upregulation contributed to OS cell invasion and migration ( $\times 100$ magnification). The cells were stained with crystal violet. OS, osteosarcoma; PART 1, prostate androgen-regulated transcript $1 .{ }^{* *}, \mathrm{P}<0.01 ;{ }^{* * *}, \mathrm{P}<0.001$. 


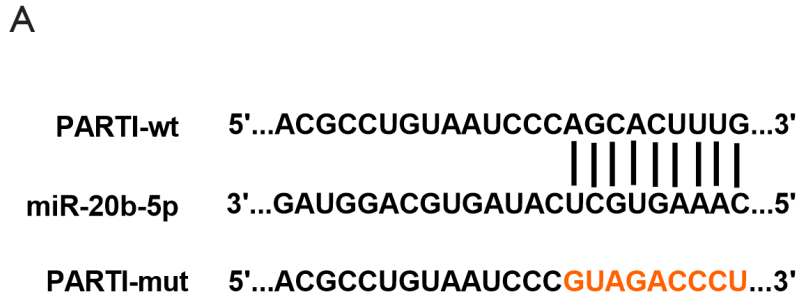

C
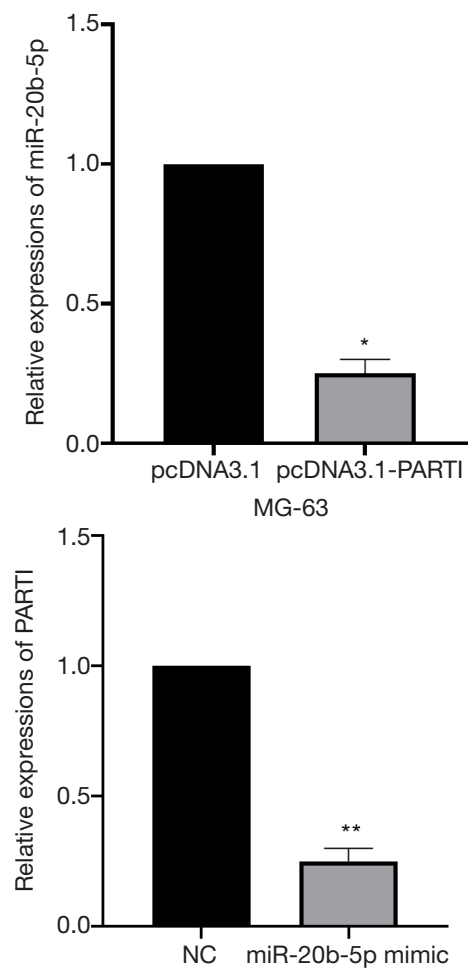

E

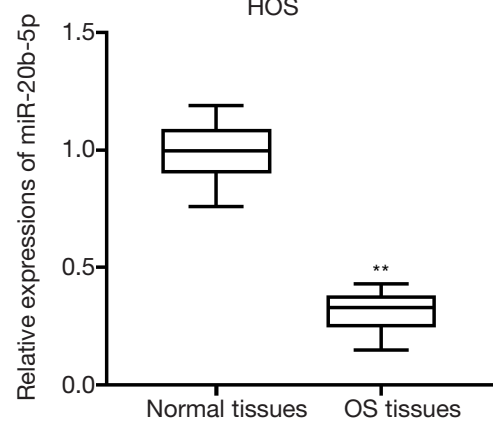

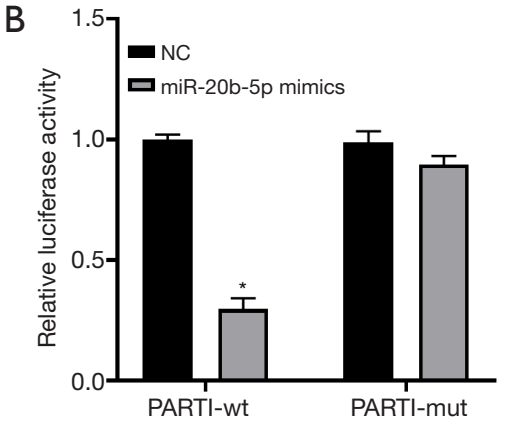
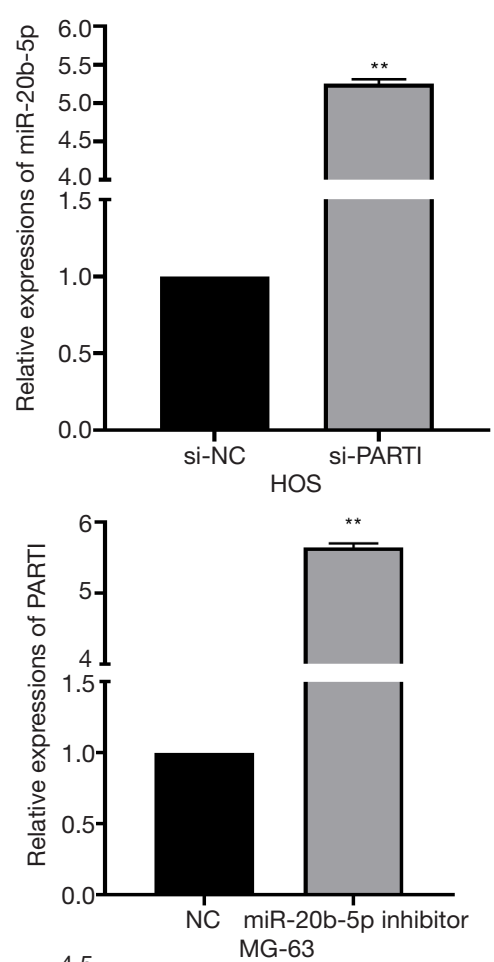

$\mathrm{F}$

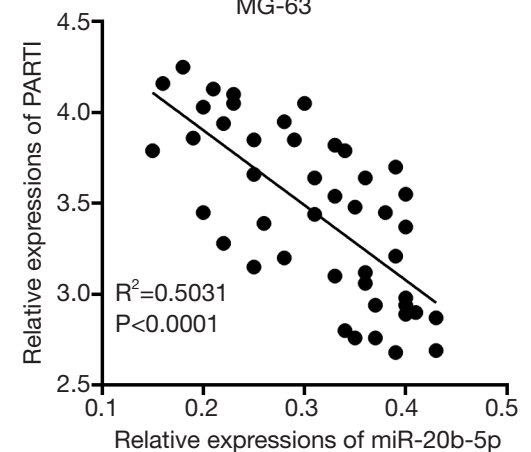

Figure 3 PART 1 acted as a sponge of miR-20b-5p in OS cells. (A) Putative binding sites of miR-20b-5p in the $3{ }^{\prime}-$ untranslated region of PART 1 were obtained from Starbase. (B) Relative luciferase activity of OS cells transfected with PART 1-wt/mut reporter plasmid and miR20b-5p mimic. (C,D) The regulatory relationship of PART 1 and miR-20b-5p was confirmed by RT-qPCR analysis. (E) Downregulated miR-20b-5p expression was identified in OS tissues. (F) A negative correlation between the expressions of PART 1 and miR-20b-5p in OS tissues was confirmed. OS, osteosarcoma; PART 1, prostate androgen-regulated transcript $1 .{ }^{*}, \mathrm{P}<0.05 ;{ }^{* *}, \mathrm{P}<0.01$. 

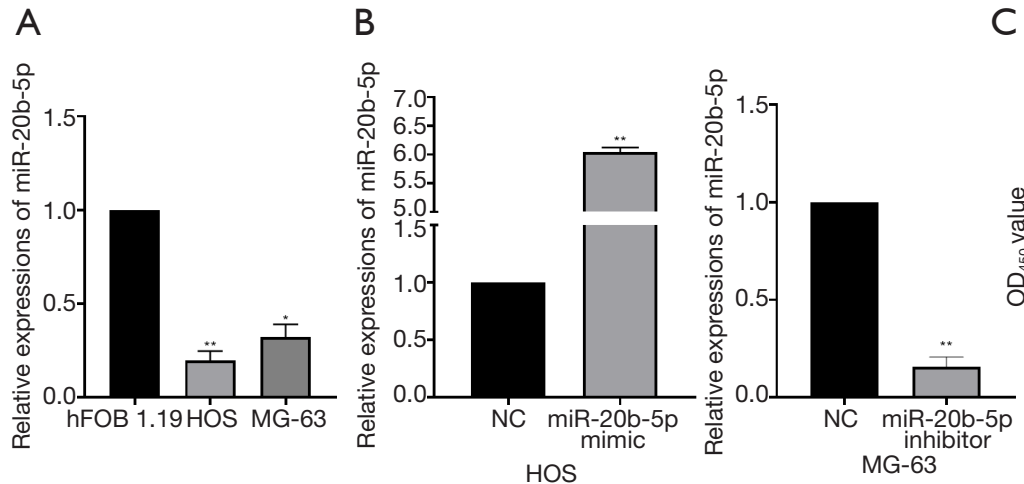

C

$\mathrm{D}$
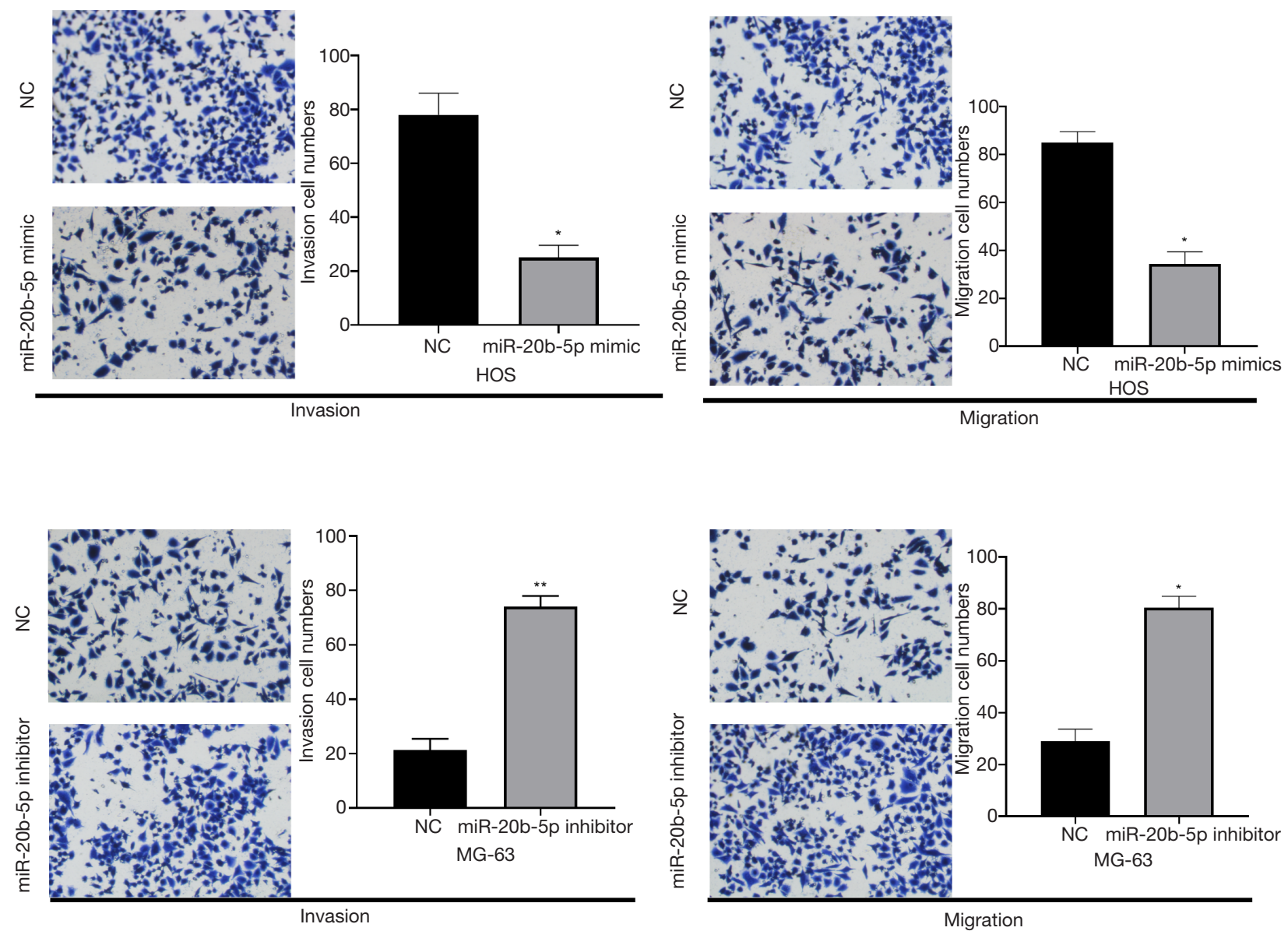

Figure 4 miR-20b-5p inhibited the proliferation, invasion, and migration in OS cells. (A) miR-20b-5p was downregulated in OS cells. (B) miR-20b-5p was efficiently overexpressed and silenced by miR-20b-5p mimics and inhibitor, respectively. (C, D) miR-20b-5p inhibited the proliferation, invasion, and migration in OS cells. The cells were stained with crystal violet in transwell assay ( $\times 100$ magnification). OS, osteosarcoma. *, $\mathrm{P}<0.05 ;{ }^{* *}, \mathrm{P}<0.01$; ***, $\mathrm{P}<0.001$. 
A
BAMBI-wt $\quad 5^{\prime}$...UUCUGCUGGACAGGAGCACUUUA...3' |IIIIIIII

miR-20b-5p $\quad 3^{\prime} \ldots$...GAUGGACGUGAUACUCGUGAAAC...5'

BAMBI-mut $\quad 5^{\prime}$...UUCUGCUGGACAGACAUCUGGGA...3'

C

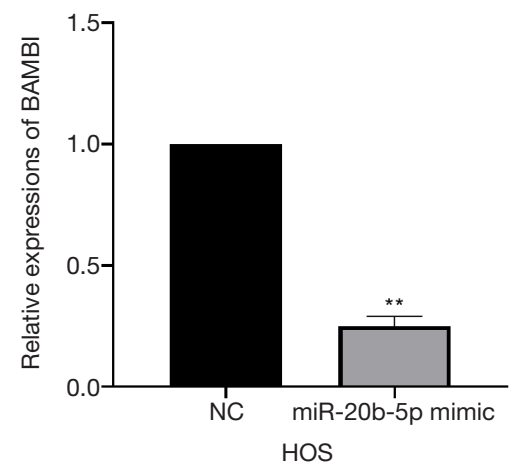

$E$

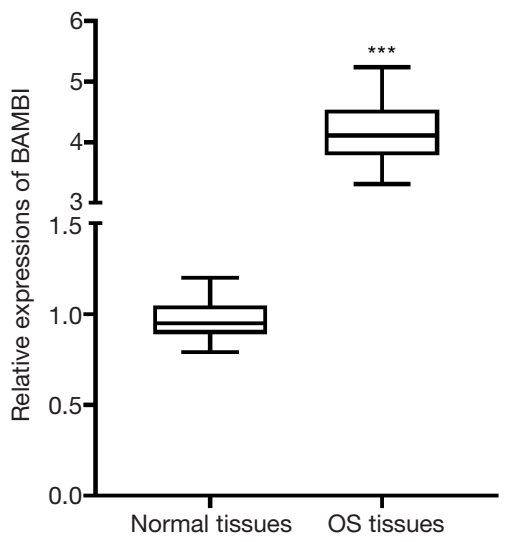

B

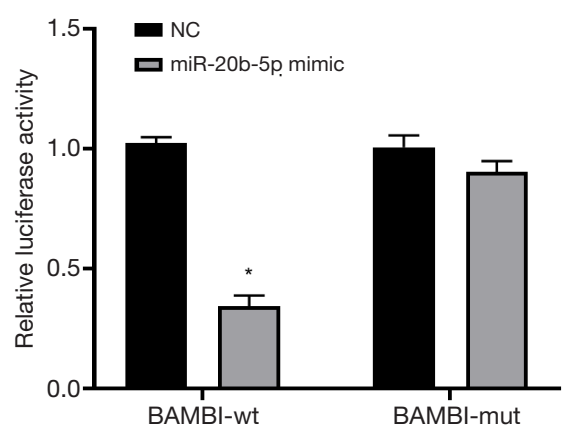

D

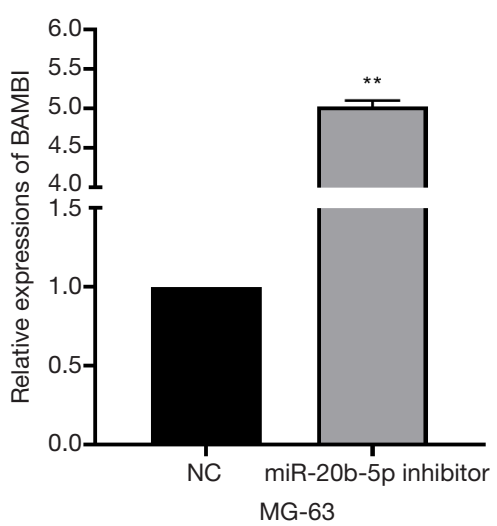

$\mathrm{F}$

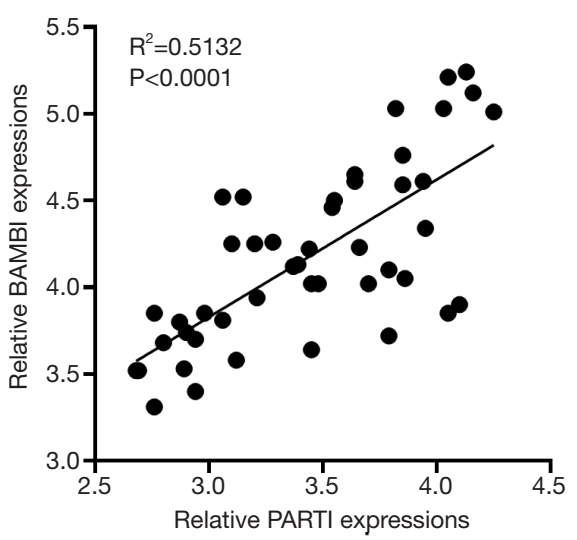

Figure 5 BAMBI served as a target of miR-20b-5p in OS cells. (A) Putative binding sites of miR-20b-5p in the 3'-UTR of BAMBI were predicted by TargetScan. (B) miR-20b-5p mimic significantly decreased the relative luciferase activity of BAMBI-wt reporter plasmid. (C,D) BAMBI expression was regulated by miR-20b-5p in OS cells. (E,F) High BAMBI expression was identified in OS tissues, and was positively correlated with PART 1 expression. OS, osteosarcoma. *, $\mathrm{P}<0.05 ;{ }^{* *}, \mathrm{P}<0.01$; ***, $\mathrm{P}<0.001$. 
A

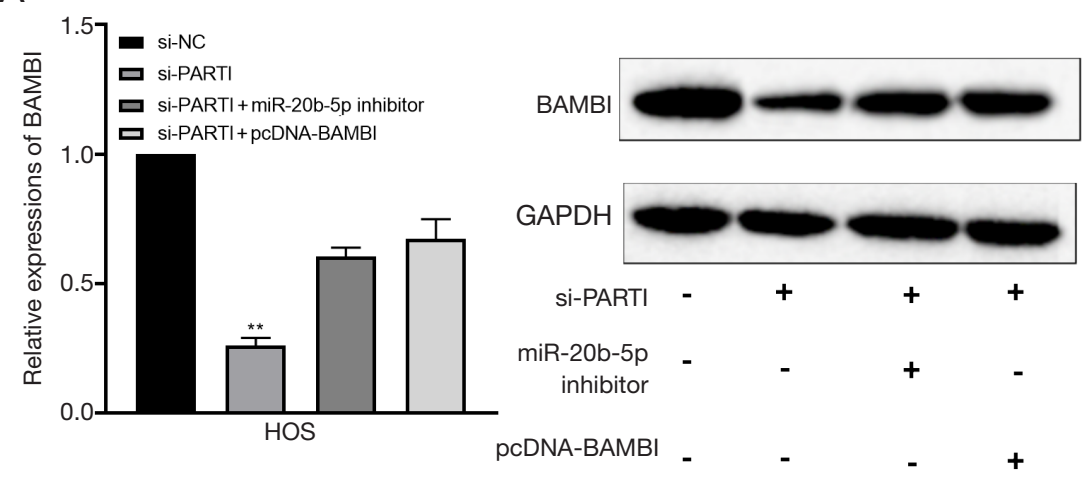

C

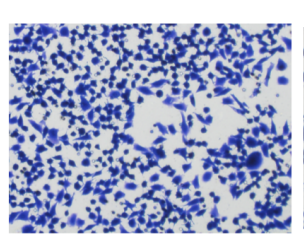

Si-PARTI

miR-20b-5p

inhibitor

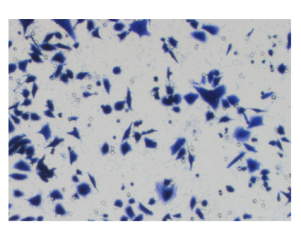

$+$

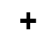

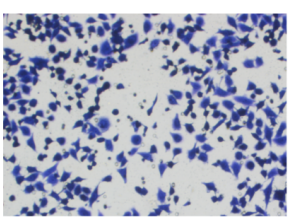

$+$

$+$

pcDNA-BAMBI

B
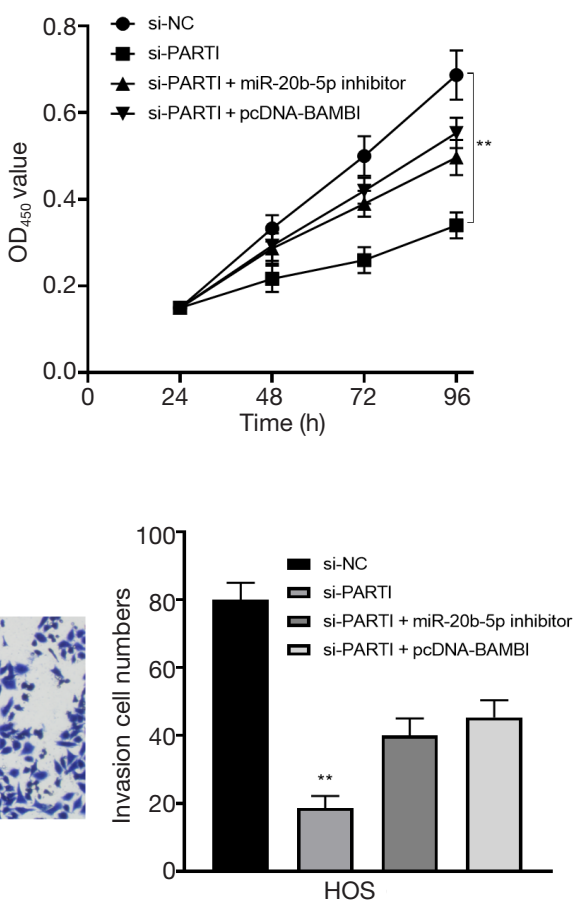

HOS

D

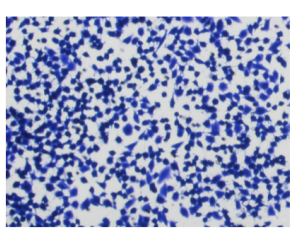

si-PARTI

miR-20b-5p

inhibitor

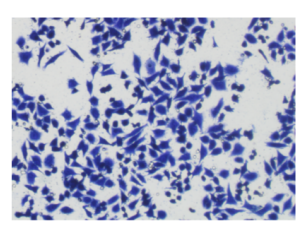

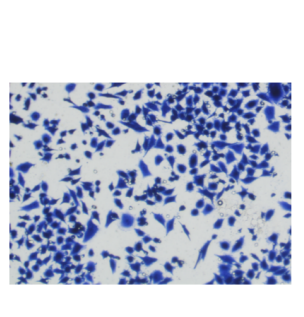

$+$

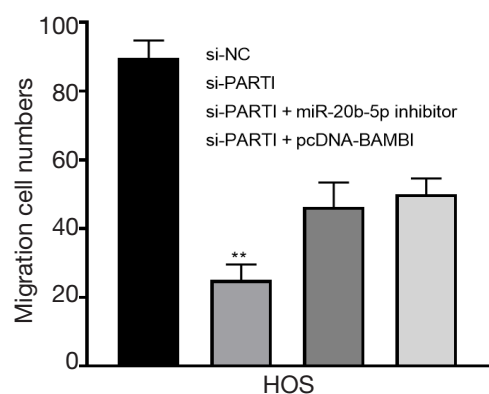

pcDNA-BAMBI

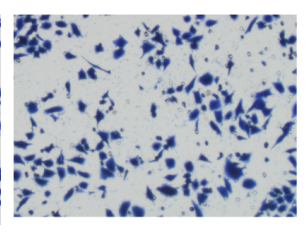

$+$

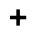

$+$

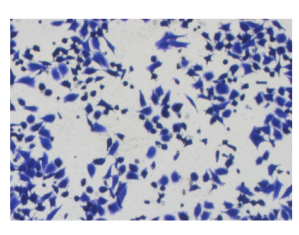

Migration

Figure 6 PART 1 promoted OS tumorigenesis by sponging miR-20b-5p to upregulate BAMBI. (A) BAMBI expression was silenced by siPART 1, and this reduction could be PART 1 ally reversed by miR-20b-5p inhibitor or pcDNA-BAMBI. (B,C,D) miR-20b-5p inhibitor or pc DNA-BAMBI could reverse the inhibitory effects of si-PART 1 in OS cell proliferation, invasion, and migration. The cells were stained with crystal violet in transwell assay ( $\times 100$ magnification). OS, osteosarcoma; PART 1 , prostate androgen-regulated transcript $1 .{ }^{* *}, \mathrm{P}<0.01$. 
on OS cell proliferation, migration, and invasion could be dramatically attenuated by miR-20b-5p silencing or BAMBI overexpression (Figure 6B,C,D). All of these data revealed PART 1 to be a modulator of OS cell malignancy through its sponging of miR-20b-5p to promote BAMBI expression.

\section{Discussion}

OS is a common bone malignancy in adolescents and teens. Tumor recurrence and metastasis are 2 primary factors for the high mortality rate of OS (22). There is mounting evidence showing that lncRNA is important in a variety of biological cellular processes $(23,24)$. Previous studies have indicated that dysregulation of lncRNAs is correlated with tumor progression and patient outcomes (25). Recently, lncRNAs have been found to play crucial roles in OS. For instance, Ding et al. found that CRNDE (colorectal neoplasia differentially expressed) facilitated OS cell proliferation, invasion, and EMT via the Wnt/betacatenin signaling pathway, following activation by SP1 (26). Furthermore, Cui et al. found that TMPO antisense RNA 1 promoted OS tumorigenesis by regulating the miR-199a$5 \mathrm{p} / \mathrm{WNT} 7 \mathrm{~B}$ axis (27). Also, a study by Zhu et al. showed that PCAT6 promoted OS progression by sponging miR185-5p and activating the transforming growth factor beta signaling pathway (28). Yet, the impact of PART 1 on the biological behavior of OS cells has remained unclear. Therefore, the aim of the present study was to elucidate the roles and mechanisms of PART 1 in OS.

LncRNA PART 1 is known as an androgen-regulated and prostate-specific gene (29). PART 1 overexpression in the prostate gland has been confirmed as being related to prostate tumor initiation (30). In recent years, aberrant PART 1 expression has also been confirmed in other tumors. For instance, Xuan et al.'s study indicated PART 1 was an independent predictor of prognosis in glioma patients (31), while Zhou et al. found that PART 1 regulated colorectal cancer via activation of the $\mathrm{Wnt} /$ beta-catenin pathway and regulation of miR-150-5p/miR-520h/CTNNB1 (32). Moreover, Zhu et al. found that PART 1 contributed to non-small cell lung cancer progression via the JAK-STAT signaling pathway (33). In our study, we found that PART 1 was upregulated in OS, with its overexpression promoting the viability, invasion, and migration of OS cells.

Accumulating studies have demonstrated that lncRNAs may serve as ceRNAs in carcinogenesis. ceRNAs can sponge miRNAs, reducing their binding to the target genes and thereby modulating their expression (34). In our study,
miR-20b-5p, which was predicted as a target of PART $1, \quad 384$ was found at low levels in OS tissues and cells. Further 385 investigation of the potential mechanisms indicated that 386 BAMBI served as a direct target of miR-20b-5p in OS cells. 387 PART 1 was found to act as a promoter of OS tumorigenesis 388 by sponging miR-20b-5p to upregulate BAMBI. 389

In conclusion, the data of the present study revealed that high levels of PART 1 and low levels of miR-20b-5p are expressed in OS. PART 1 upregulation notably contributed to OS cell proliferation and cell mobility. Further study showed that the anti-OS functions of PART 1 were exerted via its sponging of miR-20b-5p to up-regulate BAMBI. Our findings may provide novel diagnostic markers for OS and enrich our knowledge of OS progression.

\section{Acknowledgments}

Funding: This work was supported by grants from the National Natural Science Foundation of China (81702671), Jiangxi Provincial Natural Science Foundation (20202BABL216047, 20171BAB205031), and the Department of Science and Technology of Jiangxi Province (S2019ZPYFB2011).

\section{Footnote}

Reporting Checklist: The authors have completed the MDAR reporting checklist. Available at http://dx.doi.org/10.21037/ atm-21-658

Data Sharing Statement: Available at http://dx.doi. org/10.21037/atm-21-658

Conflicts of Interest: All authors have completed the ICMJE uniform disclosure form (available at http://dx.doi. org/10.21037/atm-21-658). The authors have no conflicts of interest to declare.

Ethical Statement: The authors are accountable for all aspects of the work in ensuring that questions related to the accuracy or integrity of any part of the work are appropriately investigated and resolved. All patients signed a written informed consent form. All procedures in our study were carried out in accordance with the Helsinki Declaration (as revised in 2013). The study was approved by the Ethics Committee Board of our Hospital.

Open Access Statement: This is an Open Access article 
distributed in accordance with the Creative Commons Attribution-NonCommercial-NoDerivs 4.0 International License (CC BY-NC-ND 4.0), which permits the noncommercial replication and distribution of the article with the strict proviso that no changes or edits are made and the original work is properly cited (including links to both the formal publication through the relevant DOI and the license). See: https://creativecommons.org/licenses/by-nc-nd/4.0/.

\section{References}

1. Oh JY, Kim EH, Lee YJ, et al. Synergistic Autophagy Effect of miR-212-3p in Zoledronic Acid-Treated In Vitro and Orthotopic In Vivo Models and in Patient-Derived Osteosarcoma Cells. Cancers (Basel) 2019;11:1812.

2. Friebele JC, Peck J, Pan X, et al. Osteosarcoma: A MetaAnalysis and Review of the Literature. Am J Orthop (Belle Mead NJ) 2015;44:547-53.

3. Jiang Y, Wang X, Cheng Y, et al. Associations between inflammatory gene polymorphisms (TNF-alpha 308G/ A, TNF-alpha 238G/A, TNF-beta 252A/G, TGFbeta1 29T/C, IL-6 174G/C and IL-10 1082A/G) and susceptibility to osteosarcoma: a meta-analysis and literature review. Oncotarget 2017;8:97571-83.

4. Qi L, Ren X, Liu Z, et al. Predictors and Survival of Patients with Osteosarcoma After Limb Salvage versus Amputation: A Population-Based Analysis with Propensity Score Matching. World J Surg 2020;44:2201-10.

5. Zhang Y, Wang F, Wang L, et al. MiR-363 suppresses cell migration, invasion, and epithelial-mesenchymal transition of osteosarcoma by binding to NOB1. World J Surg Oncol 2020;18:83.

6. Yu WX, Lu C, Wang B, et al. Effects of rapamycin on osteosarcoma cell proliferation and apoptosis by inducing autophagy. Eur Rev Med Pharmacol Sci 2020;24:915-21.

7. Zhang Y, Weng Q, Chen J, et al. Morusin inhibited human osteosarcoma via PI3K-AKT signaling pathway. Curr Pharm Biotechnol 2020;21:1402-9.

8. Zhou Y, Li X, Yang H. LINC00612 functions as a ceRNA for miR-214-5p to promote the proliferation and invasion of osteosarcoma in vitro and in vivo. Exp Cell Res 2020;392:112012.

9. Luo M, Liang C. LncRNA LINC00483 promotes gastric cancer development through regulating MAPK1 expression by sponging miR-490-3p. Biol Res 2020;53:14.

10. Anastasiadou E, Jacob LS, Slack FJ. Non-coding RNA networks in cancer. Nat Rev Cancer 2018;18:5-18.

11. Qian C, Liao CH, Tan BF, et al. LncRNA PROX1-AS1 promotes proliferation, invasion, and migration in prostate cancer via targeting miR-647. Eur Rev Med Pharmacol Sci 2020;24:2938-44.

12. Qiao Z, Dai H, Zhang Y, et al. LncRNA NCK1AS1 Promotes Cancer Cell Proliferation and Increase Cell Stemness in Urinary Bladder Cancer Patients by Downregulating miR-143. Cancer Manag Res 2020;12:1661-8.

13. Qin CX, Yang XQ, Jin GC, et al. LncRNA TSLNC8 inhibits proliferation of breast cancer cell through the miR-214-3p/FOXP2 axis. Eur Rev Med Pharmacol Sci 2019;23:8440-8.

14. Sun Y, Zhou Q, Li J, et al. LncRNA RP11-422N16.3 Inhibits Cell Proliferation and EMT, and Induces Apoptosis in Hepatocellular Carcinoma Cells by Sponging miR-23b-3p. Onco Targets Ther 2019;12:10943-61.

15. Misawa A, Orimo H. lncRNA HOTAIR Inhibits Mineralization in Osteoblastic Osteosarcoma Cells by Epigenetically Repressing ALPL. Calcif Tissue Int 2018;103:422-30.

16. Zhang N, Meng X, Mei L, et al. LncRNA DLX6AS1 promotes tumor proliferation and metastasis in osteosarcoma through modulating miR-641/HOXA9 signaling pathway. J Cell Biochem 2019. [Epub ahead of print]. doi: 10.1002/jcb.28426.

17. $\mathrm{Lu} \mathrm{C}, \mathrm{Li} Z, \mathrm{Hu}$, et al. LncRNA PART-1 targets TGFBR2/Smad3 to regulate cell viability and apoptosis of chondrocytes via acting as miR-590-3p sponge in osteoarthritis. J Cell Mol Med 2019;23:8196-205.

18. Pu J, Tan C, Shao Z, et al. Long Noncoding RNA PART1 Promotes Hepatocellular Carcinoma Progression via Targeting miR-590-3p/HMGB2 Axis. Onco Targets Ther 2020;13:9203-11.

19. Iorio MV, Croce CM. microRNA involvement in human cancer. Carcinogenesis 2012;33:1126-33.

20. Alvarez-Garcia I, Miska EA. MicroRNA functions in animal development and human disease. Development 2005;132:4653-62.

21. Patil SL, Palat A, Pan Y, et al. MicroRNA-509-3p inhibits cellular migration, invasion, and proliferation, and sensitizes osteosarcoma to cisplatin. Sci Rep 2019;9:19089.

22. Basile P, Greengard E, Weigel B, et al. Prognostic Factors for Development of Subsequent Metastases in Localized Osteosarcoma: A Systematic Review and Identification of Literature Gaps. Sarcoma 2020;2020:7431549.

23. Kawasaki Y, Miyamoto M, Oda T, et al. The novel lncRNA CALIC upregulates AXL to promote colon cancer metastasis. EMBO Rep 2019;20:e47052. 
24. Carlevaro-Fita J, Lanzos A, Feuerbach L, et al. Cancer LncRNA Census reveals evidence for deep functional conservation of long noncoding RNAs in tumorigenesis. Commun Biol 2020;3:56.

25. Wang W, Xie Y, Chen F, et al. LncRNA MEG3 acts a biomarker and regulates cell functions by targeting ADAR1 in colorectal cancer. World J Gastroenterol 2019;25:3972-84.

26. Ding Q, Mo F, Cai X, et al. LncRNA CRNDE is activated by SP1 and promotes osteosarcoma proliferation, invasion, and epithelial-mesenchymal transition via $\mathrm{Wnt} /$ beta-catenin signaling pathway. J Cell Biochem 2020;121:3358-71.

27. Cui H, Zhao J. LncRNA TMPO-AS1 serves as a ceRNA to promote osteosarcoma tumorigenesis by regulating miR-199a-5p/WNT7B axis. J Cell Biochem 2020;121:2284-93.

28. Zhu C, Huang L, Xu F, et al. LncRNA PCAT6 promotes tumor progression in osteosarcoma via activation of TGFbeta pathway by sponging miR-185-5p. Biochem Biophys Res Commun 2020;521:463-70.

29. Sidiropoulos M, Chang A, Jung K, et al. Expression and regulation of prostate androgen regulated transcript-1

Cite this article as: Pan Z, Mo F, Liu H, Zeng J, Huang K, Huang S, Cao Z, Xu X, Xu J, Liu T, Huang J. LncRNA prostate androgen-regulated transcript 1 (PART 1) functions as an oncogene in osteosarcoma via sponging miR-20b-5p to upregulate BAMBI. Ann Transl Med 2021;9(6):488. doi: 10.21037/atm-21-658
(PART-1) and identification of differential expression in 551 prostatic cancer. Br J Cancer 2001;85:393-7. 552

30. Lin B, White JT, Ferguson C, et al. PART-1: a novel 553 human prostate-specific, androgen-regulated gene that 554 maps to chromosome 5q12. Cancer Res 2000;60:858-63. 555

31. Xuan C, Jin M, Wang L, et al. PART1 and hsa-miR- 556 429-Mediated SHCBP1 Expression Is an Independent 557 Predictor of Poor Prognosis in Glioma Patients. Biomed 558 Res Int 2020;2020:1767056.

32. Zhou T, Wu L, Ma N, et al. LncRNA PART1 regulates 560 colorectal cancer via targeting miR-150-5p/miR-520h/ 561 CTNNB1 and activating Wnt/beta-catenin pathway. Int J 562 Biochem Cell Biol 2020;118:105637. 563

33. Zhu D, Yu Y, Wang W, et al. Long noncoding RNA 564 PART1 promotes progression of non-small cell lung 565 cancer cells via JAK-STAT signaling pathway. Cancer Med 566 2019;8:6064-81.

34. Tay Y, Rinn J, Pandolfi PP. The multilayered 568 complexity of ceRNA crosstalk and competition. Nature $\quad 569$ 2014;505:344-52. 570 